\title{
Hemorrhagic Ascites Is Associated With Reduced Survival in Cirrhosis: A Systematic Review and Meta-Analysis
}

\author{
Umair Iqbal $^{\mathrm{a}}$, Zohaib Ahmed ${ }^{\mathrm{b}}$, Hafsa Anwar ${ }^{\mathrm{c}}$, Nihit M. Shah ${ }^{\mathrm{a}}$, Wade Lee ${ }^{\mathrm{b}}$, Ali Nawras ${ }^{\mathrm{d}}$, \\ Harshit S. Khara ${ }^{a}$, Aijaz Ahmed ${ }^{\mathrm{e}}$, Sandeep Khurana, ${ }^{\mathrm{a}}$,
}

\begin{abstract}
Background: Hemorrhagic ascites is characterized as red blood cell count greater than $10,000 / \mathrm{mm}^{3}$. In cirrhosis, ascites is an event of decompensation, and associated with poor prognosis. However, significance of hemorrhagic ascites is unclear. We conducted a systematic review and meta-analysis to evaluate the significance of hemorrhagic ascites in cirrhotic patients.
\end{abstract}

Methods: We conducted a systematic search in Embase, MEDLINE, Cochrane Central Register of Controlled Trials, the World Health Organization (WHO) International Clinical Trial Registry, and Web of Science Core Collection to identify studies till March 2021, which, in patients with cirrhosis, compared outcomes amongst those with hemorrhagic ascites to those with non-hemorrhagic ascites. The primary outcome was 3-year mortality, and secondary outcomes were acute kidney injury (AKI), hepatic encephalopathy (HE), spontaneous bacterial peritonitis (SBP) and portal vein thrombosis (PVT).

Results: Four studies, with 2,058 cirrhosis patients, were included. Among these, 1,488 patients had non-hemorrhagic ascites and 570 had hemorrhagic ascites. We observed no significant differences in $\mathrm{AKI}$ (odds ratio $(\mathrm{OR})=2.55$; confidence interval $(\mathrm{CI})$ : $0.58-11.24$ ), $\mathrm{HE}(\mathrm{OR}=2.52$; CI: $0.70-9.05), \mathrm{SBP}(\mathrm{OR}=1.66$; CI: $0.12-22.83)$ and PVT $(\mathrm{OR}=0.99$; CI: $0.71-1.39)$. Intensive care unit (ICU) stay was significantly higher in patients with hemorrhagic ascites compared to those with non-hemorrhagic ascites $(\mathrm{OR}=1.79$; CI: 1.37 - 2.36; $\mathrm{I}^{2}=56 \%$ ). Pooled 3-year mortality was significantly higher in those with hemorrhagic (72.5\% (CI: $68.2-76.4 \%)$ ) when compared to

Manuscript submitted November 16, 2021, accepted January 20, 2022 Published online February 17, 2022

aDepartment of Gastroenterology and Hepatology, Geisinger Medical Center, Danville, PA, USA

bepartment of Internal Medicine, University of Toledo, Toledo, OH, USA

'Department of Internal Medicine, Capital Health Regional Medical Center, Trenton, NJ, USA

${ }^{\mathrm{d}}$ Department of Gastroenterology and Hepatology, University of Toledo, Toledo, OH, USA

eDepartment of Gastroenterology and Hepatology, Stanford Medical Center, Palo Alto, CA, USA

${ }^{f}$ Corresponding Author: Sandeep Khurana, Department of Gastroenterology and Hepatology, Geisinger Medical Center, Danville, PA 17822, USA.

Email: skhurana@geisinger.edu

doi: https://doi.org/10.14740/gr1485 non-hemorrhagic ascites (57.9\% (CI: 55.2-60.6\%) $(\mathrm{OR}=2.17$; CI: $1.71-2.74)$ with low heterogeneity $\left(\mathrm{I}^{2}=15 \%\right)$.

Conclusions: In patients with cirrhosis, hemorrhagic ascites is a poor prognostic marker, which is associated with increased ICU stay and mortality. Prospective studies are needed to further evaluate significance of hemorrhagic ascites in patients with cirrhosis.

Keywords: Hepatic encephalopathy; Spontaneous bacterial peritonitis; Portal vein thrombosis; Acute kidney injury; Intensive care unit

\section{Introduction}

Cirrhosis is advanced hepatic fibrosis characterized by hepatic parenchymal distortion and regenerative nodule formation; it is associated with multiple complications, and sometimes, liver transplantation is the only cure for these patients [1]. Cirrhosis is the 11th leading cause of death with 44,478 deaths in 2017 , and accounts for $2.4 \%$ deaths globally $[2,3]$. Some of the common complications of cirrhosis include ascites, variceal bleeding, spontaneous bacterial peritonitis (SBP), hepatic encephalopathy (HE), hepatocellular carcinoma, and hepatopulmonary syndrome [4]. Ascites, a pathological fluid buildup in the peritoneal cavity, is one of the most common complications, with an estimated prevalence of $10 \%$ [5]. In cirrhotic patients, development of portal hypertension is the first step toward fluid retention and ascites. Patients without portal hypertension usually do not develop ascites or edema [6, 7]. Cirrhosis also induces disturbances in systemic vasoactive factors and intrarenal factors which play a major role in the formation of ascites. In advanced cirrhosis, patients often have an inability to maintain the extracellular fluid (ECF) volume within normal limits, resulting in a large amount of fluid accumulation in the peritoneal and pleural cavities. Changes in intrinsic renal functions especially sodium and water retention lead to an increase in ECF volume, increased total body water, and hyponatremia. Patients with cirrhosis and ascites have an increase in plasma volume and cardiac output which lead to hyperdynamic circulation. They have a marked reduction in arterial pressure and systemic vascular resistance, which then activate vasoconstrictors and anti-natriuretic systems. These marked changes cause renal vasoconstriction and reduce renal plasma flow and glomerular filtration rate (GFR). All of these 
mechanisms ultimately result in the formation of ascites [8]. Development of ascites is associated with poor prognosis and a high mortality rate, and is frequently associated with other complications such as SBP, hyponatremia and renal dysfunction [9].

Hemorrhagic ascites is described as a red blood cell (RBC) count greater than $10,000 / \mathrm{mm}^{3}$ in ascitic fluid, and it affects $5 \%$ of cirrhotic patients [10]. Limited data are available on the significance of hemorrhagic ascites in patients with cirrhosis. Therefore, to evaluate the impact of hemorrhagic ascites in patients with cirrhosis, we conducted a systematic review and meta-analysis.

\section{Materials and Methods}

\section{Search strategy}

A comprehensive search strategy was developed by an experienced health sciences librarian (WL-S) for Embase (Elsevier site), and then recreated for MEDLINE (PubMed platform), Cochrane Central Register of Controlled Trials, which includes trial registries from ClinicalTrials.gov and the World Health Organization (WHO) International Clinical Trial Registry (Wiley Cochrane Library platform), and Web of Science Core Collection (Web of Science Platform). Search terms included truncated keyword and phrase searching for the topics of hemorrhagic ascites and liver cirrhosis, with database's controlled vocabularies used when available. Search limits (when available) were used to eliminate animal studies and to exclude reviews, editorials, guidelines, prior systematic reviews, and small sample case reports from the results. No language limits were applied, and databases were searched from inception to March 5, 2021. Entire search strategies are available here (Supplementary Material 1, www.gastrores.org). Results were exported to EndNote X9.3 (Clarivate, Philadelphia, PA, USA) and deduplicated by author, title, and year and by visual inspection.

\section{Statistical analysis}

Statistical analysis was conducted utilizing RevMan 5.4 and Comprehensive Meta-Analysis softwares. Fixed and randomeffects model were utilized for this meta-analysis, with point estimates, variance, and weights for each study based on the size of the study and the number of events. Pooled odds ratio (OR) was calculated for primary and secondary outcomes. The primary outcome was difference in 3-year mortality, defined as death from any cause in 3 years. Secondary outcomes were difference in occurrence of acute kidney injury (AKI), HE, spontaneous bacterial peritonitis (SBP) and portal vein thrombosis (PVT). Heterogeneity among the studies was evaluated using the $\mathrm{I}^{2}$ test. A value of $\mathrm{I}^{2}$ of $0-25 \%$ represented insignificant heterogeneity, 26-50\% represented low heterogeneity, $51-75 \%$ represented moderate heterogeneity, and $>75 \%$ represented high heterogeneity. The $\alpha$ was set at 0.05 ; $\mathrm{P}$ value $<0.05$ was considered statistically significant. We performed quality as- sessment of every study using Newcastle Ottawa quality assessment scale [11]. Our meta-analysis was conducted in accordance with the PRISMA guidelines [12].

The Institutional Review Board approval is not applicable for this study.

\section{Results}

The initial search strategy revealed 56 studies. After removal of duplicates, 36 studies underwent title review, of which 17 underwent full-text review. Thirteen studies were excluded after full-text review due to lack of reporting of outcomes of interest and insufficient data. Figure 1 elaborates the systematic literature search of our study. Four studies, including 2,058 cirrhosis patients, met our inclusion criteria [10, 13-15]. Three studies were rated as good quality and one study was of fair quality. Amongst these, 1,488 patients had non-hemorrhagic ascites, and 570 had hemorrhagic ascites. MELD and Child-Pugh scores were significantly higher in patients with hemorrhagic ascites. Table $1[10,13-15]$ reports baseline characteristics of patients, including MELD scores, Child-Pugh scores, demographics, etiology of cirrhosis and cause of hemorrhagic ascites. There was no statistically significant difference in outcomes of AKI (OR $=2.55$; confidence interval $\left.(\mathrm{CI}): 0.58-11.24 ; \mathrm{I}^{2}=97 \%\right)$, $\mathrm{HE}$ $\left(\mathrm{OR}=2.52\right.$; CI: $\left.0.70-9.05 ; \mathrm{I}^{2}=93 \%\right), \mathrm{SBP}(\mathrm{OR}=1.66 ; \mathrm{CI}$ : 0.12 - 22.83; $\left.\mathrm{I}^{2}=99 \%\right)$ and PVT (OR = 0.99; CI: 0.71 - 1.39; $\mathrm{I}^{2}$ $=0$ ) between patients who had hemorrhagic ascites compared to non-hemorrhagic ascites (Figs. 2-5).

The need for intensive care unit (ICU) stay was significantly higher in patients who had hemorrhagic ascites compared to those who had non-hemorrhagic ascites $(\mathrm{OR}=$ 1.79; CI: 1.37 - 2.36; $\mathrm{I}^{2}=56 \%$ ) (Fig. 6). Three-year mortality was significantly higher in patients who had hemorrhagic ascites (pooled 3-year mortality of 72.5\% (CI: 68.2$\left.76.4 \% ; \mathrm{I}^{2}=96 \%\right)$ ) compared to non-hemorrhagic ascites with pooled mortality (pooled 3-year mortality of $57.9 \%$ (CI: 55.2-60.6\%; $\left.\mathrm{I}^{2}=98.9 \%\right)(\mathrm{OR}=2.17$; CI: $1.71-2.74)$ with heterogeneity $\left(\mathrm{I}^{2}=15 \%\right)$ (Fig. 7). Publication bias was deferred as numbers of studies included in the meta-analysis were less than 10 .

\section{Discussion}

To the best of our knowledge and literature search, this is the first systematic review and meta-analysis that comprehensively assess the significance of hemorrhagic ascites and its impact on prognosis in patients with cirrhosis. Some studies reported that hemorrhagic ascites is a poor prognostic indicator due to its association with an elevated risk of encephalopathy, acute renal failure, and a high mortality rate. Yildiz et al revealed that patient with hemorrhagic ascites had a higher rate of hepatorenal syndrome, spontaneous bacterial peritonitis, and ICU admissions [14]. Urrunaga et al showed that patients with hemorrhagic ascites have a higher risk of ICU admissions, AKI, and death than patients with portal hypertension and non-hemorrhagic ascites [10]. Desitter et al reported 


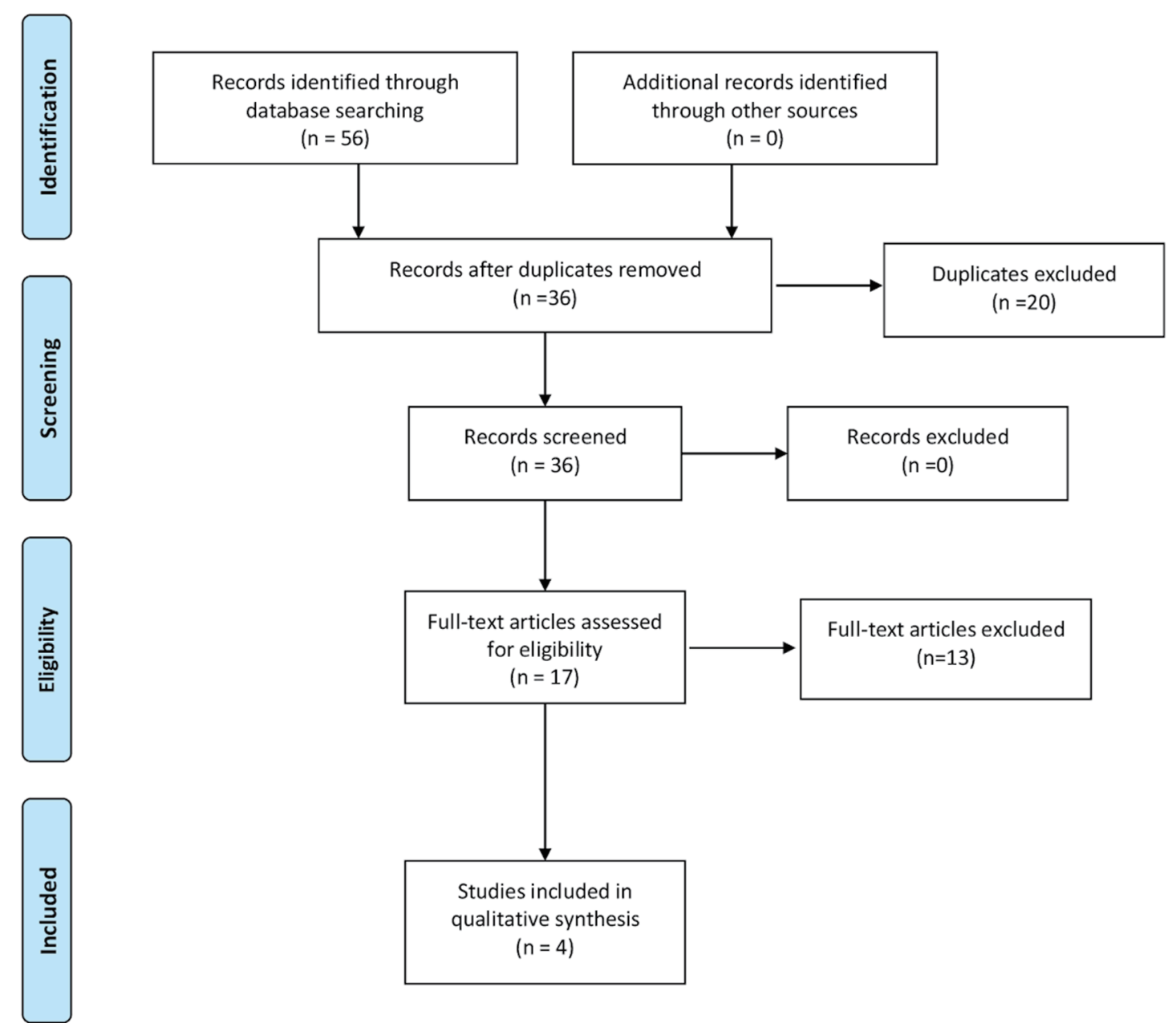

Figure 1. Literature review process.

that hemorrhagic ascites impacted overall survival with increased mortality compared to non-hemorrhagic ascites [13]. On the other hand, Naqvi et al showed higher incidence of SBP and AKI in patients with non-hemorrhagic ascites compared to patients with hemorrhagic ascites. However, mortality was significantly higher in patients with hemorrhagic ascites [15].

While no existing mechanism has been found to understand how RBCs translocate into ascites, some theories have been suggested, including the possibility of portal hypertension [16]. It is most likely the product of one of these processes: either intra-abdominal bleeding caused by an organ, a narrow peritoneal vessel, or an abdominal cavity varix, or a rise in the portal or splenic hydrostatic pressure [15]. The increase in hydrostatic pressure may cause erythrocyte leakage from the blood vessels into the peritoneal cavity [17]. Hemorrhagic ascites may occur spontaneously or because of traumatic paracentesis. The clinical course of spontaneous hemorrhagic ascites is often less severe. Most patients complain of abdominal swelling and fatigue, but cirrhotic patients with ruptured varices or hepatocellular cancer (HCC) rupture may experience hypotension, low hemoglobin levels, and rapid ab- dominal swelling [14]

The most frequent cause of bloody ascites is traumatic paracentesis; in this situation, blood usually clots instead of nontraumatic bloody ascites, where blood is lysed and may not clot [18]. Since cirrhosis patients may have an underlying malignancy such as hepatocellular carcinoma, the occurrence of nontraumatic bloody ascites in presence of malignancy is linked to an increased risk of morbidity and mortality [10]. In most cases of hemorrhagic ascites, the underlying pathophysiology is either mass impact eroding into small vessels or intense shear stress over small vessels and lymphatics [19]. Spontaneous bleeding into the ascites typically occurs slowly and does not always result in hemodynamic instability [18].

Although our systematic search is rigorous and included all the studies available on this topic, there are some limitations to our study results. First, all the studies are retrospective observational studies which may propagate bias in the study results. Second, although the sample size of the overall metaanalysis is reasonable, the numbers of studies are limited; and further prospective studies are needed to further evaluate the significance of hemorrhagic ascites in patients with cirrhosis. 
Table 1. Baseline Characteristics of the Included Studies

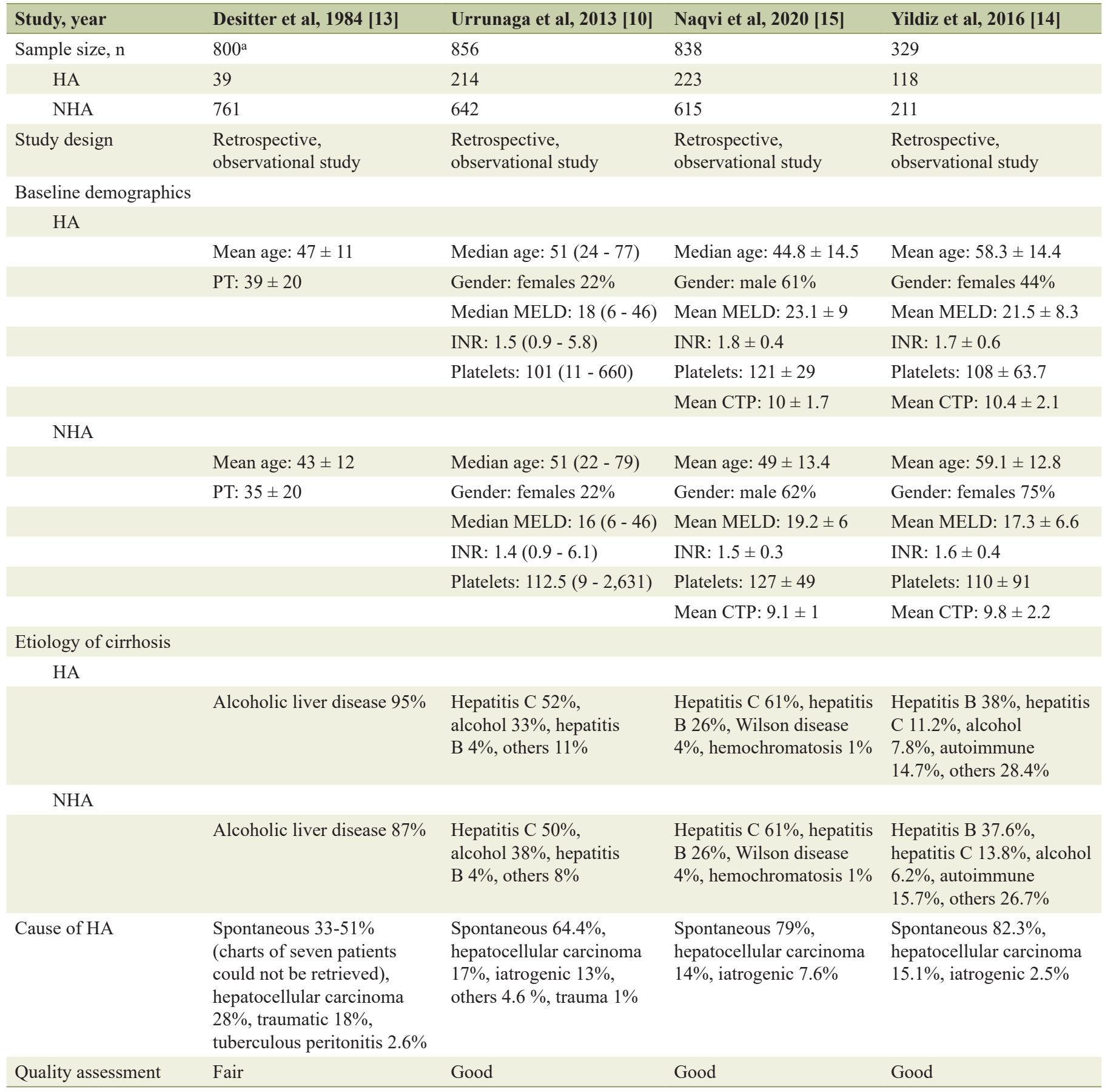

aComparative outcomes were reported in only 35 patients. NHA: non-hemorrhagic ascites; HA: hemorrhagic ascites; MELD; model for end-stage liver disease; N: total sample size; PT: prothrombin time; CTP: Child-Turcot-Pugh score; INR: international normalized ratio.

Lastly, as this is a meta-analysis, we did not have access to individual patient medical records, and the results are based on evidence reported in the individual studies.

In summary, hemorrhagic ascites appears to be associated with higher 3-year mortality in patients with cirrhosis, and is associated with an increased risk of ICU stay. It may be considered as a prognostic marker in patients with cirrhosis.
Larger prospective studies are needed to further evaluate the significance of hemorrhagic ascites in patients with cirrhosis.

\section{Supplementary Material}

Suppl 1. Entire search strategies. 


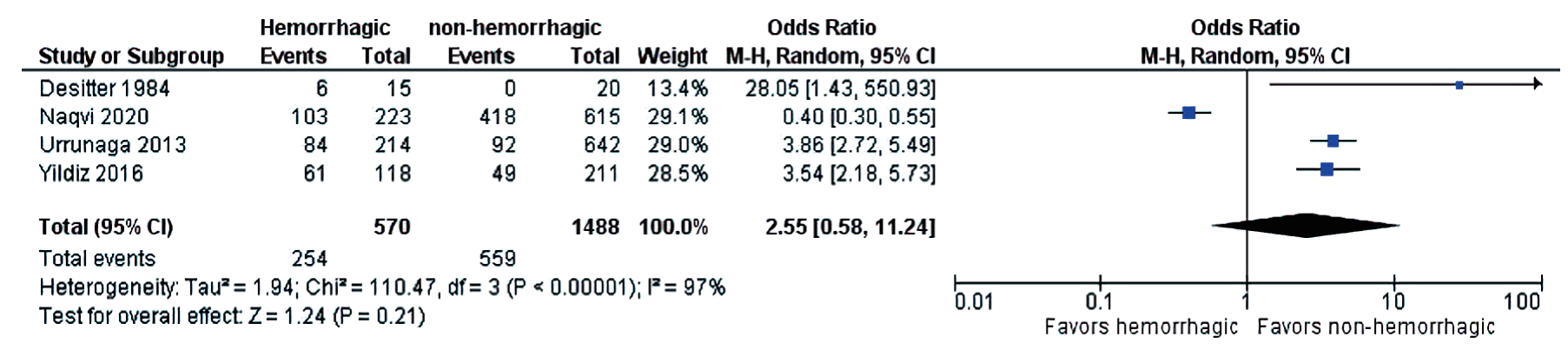

Figure 2. Difference in acute kidney injury. Cl: confidence interval.

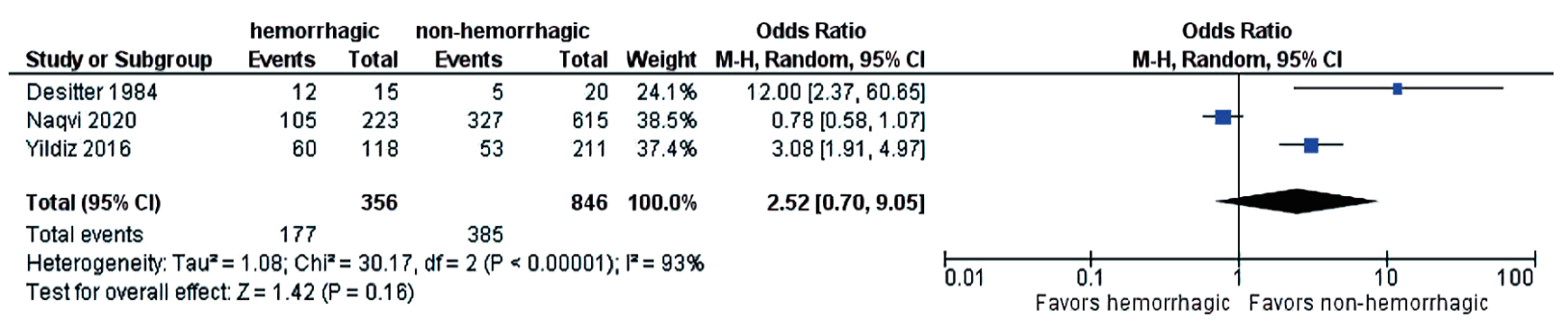

Figure 3. Difference in hepatic encephalopathy. $\mathrm{Cl}$ : confidence interval.

\section{Acknowledgments}

None to declare.

\section{Financial Disclosure}

None to declare.

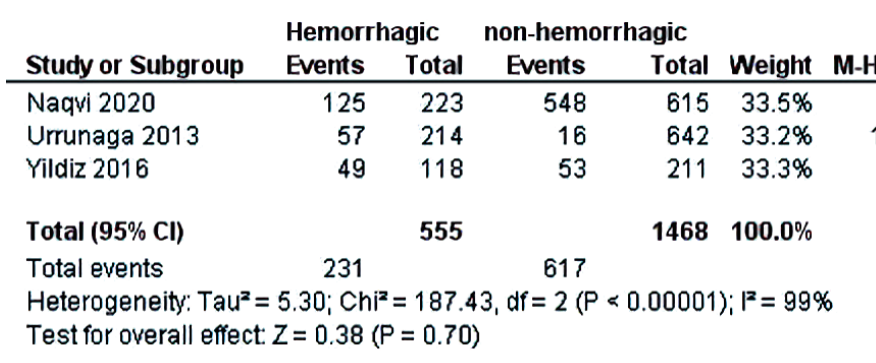

\section{Conflict of Interest}

We do not have any conflict of interest.

\section{Informed Consent}

Not applicable.

Figure 4. Difference in SBP. SBP: spontaneous bacterial peritonitis; Cl: confidence interval.

Odds Ratio

$\mathrm{H}$, Random, 95\% Cl

$0.16[0.11,0.22]$

$14.20[7.94,25.41]$

$2.12[1.31,3.42]$

$1.66[0.12,22.83]$

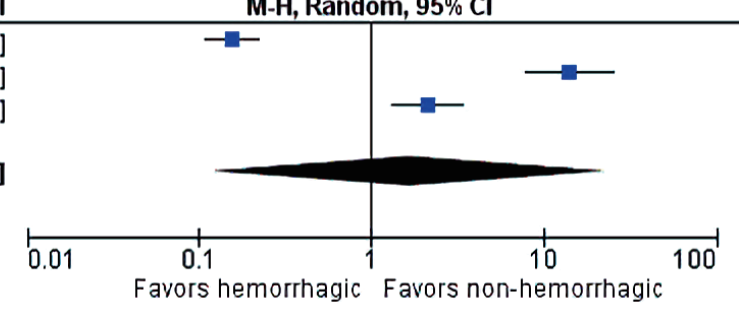

Odds Ratio $\mathrm{M}-\mathrm{H}$, Random, $95 \% \mathrm{Cl}$

non-hemorrhagic hemorrhagic Odds Ratio Odds Ratio

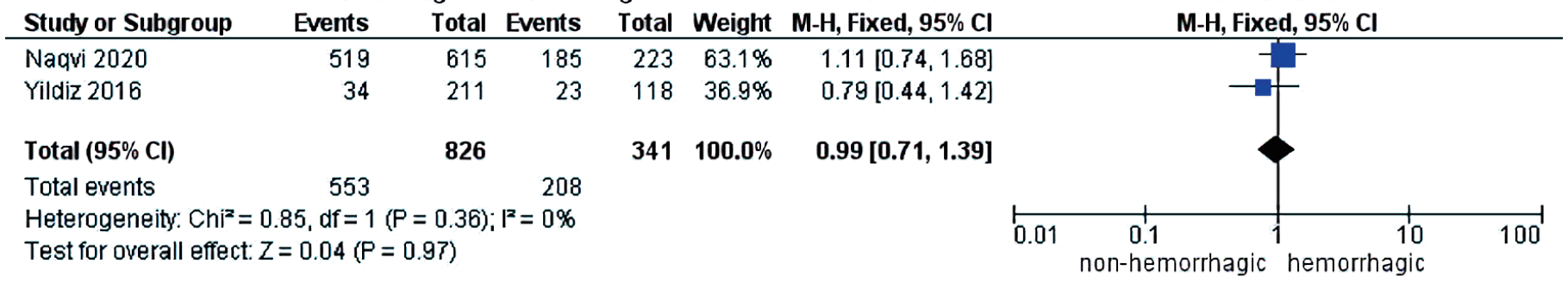

Figure 5. Difference in portal vein thrombosis. Cl: confidence interval. 


\begin{tabular}{|c|c|c|c|c|c|c|c|c|c|c|}
\hline Study or Subgroup & \multicolumn{2}{|c|}{ hemorrhagic ascites } & \multicolumn{2}{|c|}{ non-hemorrhagic ascites } & Weight & \multirow{2}{*}{$\begin{array}{c}\text { Odds Ratio } \\
\text { M-H, Fixed, 95\% Cl }\end{array}$} & \multicolumn{4}{|c|}{$\begin{array}{c}\text { Odds Ratio } \\
\text { M-H, Fixed, 95\% Cl }\end{array}$} \\
\hline Urrunaga 2013 & 68 & 214 & 150 & 642 & $68.8 \%$ & & & & & \\
\hline Yildiz 2016 & 77 & 118 & 93 & 211 & $31.2 \%$ & $2.38[1.49,3.80]$ & & & $\rightarrow-$ & \\
\hline Total $(95 \% \mathrm{Cl})$ & & 332 & & 853 & $100.0 \%$ & $1.79[1.37,2.36]$ & & & & \\
\hline Total events & 145 & & 243 & & & & & & & \\
\hline $\begin{array}{l}\text { Heterogeneity: } \mathrm{Ch}^{2}= \\
\text { Test for overall effect }\end{array}$ & $\begin{array}{l}2.28, d f=1(P \\
Z=4.20(P<\end{array}$ & $\begin{array}{l}\text { 13); }\left.\right|^{2}= \\
\text { 11) }\end{array}$ & $56 \%$ & & & & 0.01 & $\begin{array}{l}0.1 \\
\text { vors hemorrhagic }\end{array}$ & 10 & 100 \\
\hline
\end{tabular}

Figure 6. Difference in ICU stay. ICU: intensive care; CI: confidence interval.

\begin{tabular}{|c|c|c|c|c|c|c|c|c|c|c|}
\hline \multirow[b]{2}{*}{ Study or Subgroup } & \multicolumn{2}{|c|}{ hemorrhagic ascites } & \multicolumn{2}{|c|}{ non-hemorrhagic ascites } & \multirow[b]{2}{*}{ Weight } & \multirow{2}{*}{$\begin{array}{c}\text { Odds Ratio } \\
\text { M-H, Fixed, } 95 \% \mathrm{Cl}\end{array}$} & \multirow{2}{*}{\multicolumn{4}{|c|}{$\begin{array}{c}\text { Odds Ratio } \\
\text { M-H, Fixed, 95\% Cl }\end{array}$}} \\
\hline & Events & Total & Events & Total & & & & & & \\
\hline Naqvi 2020 & 185 & 223 & 449 & 615 & $40.6 \%$ & $1.80[1.22,2.66]$ & & & $\because-$ & \\
\hline Urrunaga 2013 & 126 & 214 & 250 & 642 & $51.3 \%$ & $2.25[1.64,3.08]$ & & & -1 & \\
\hline Yildiz 2016 & 110 & 118 & 168 & 211 & $8.1 \%$ & $3.52[1.59,7.77]$ & & & & \\
\hline Total $(95 \% \mathrm{Cl})$ & & 555 & & 1468 & $100.0 \%$ & $2.17[1.71,2.74]$ & & & & \\
\hline Total events & 421 & & 867 & & & & & & & \\
\hline $\begin{array}{l}\text { Heterogeneity: } \mathrm{Chi}^{2}= \\
\text { Test for overall effect }\end{array}$ & $\begin{array}{l}2.35, d f=2(f \\
Z=6.46(P \leftarrow\end{array}$ & $\begin{array}{l}\text { 31); }\left.\right|^{2}= \\
\text { j01) }\end{array}$ & $15 \%$ & & & & 0.01 & $\begin{array}{l}0.1 \\
\text { emo }\end{array}$ & $\begin{array}{r}10 \\
\text { Favors non-hemor }\end{array}$ & 100 \\
\hline
\end{tabular}

Figure 7. Difference in 3-year mortality. Cl: confidence interval.

\section{Author Contributions}

Umair Iqbal and Zohaib Ahmed were involved in writing the full manuscript. Umair Iqbal performed statistical analysis. Hafsa Anwar and Nihit Shan were involved in data collection process. Harshit S. Khara, Aijaz Ahmed, Ali Nawrad and Sandeep Khurana are the senior authors and were involved in supervising the study and finalizing the manuscript. Wade Lee performed the systematic literature search of the metaanalysis. All authors agreed to the final version of the manuscript.

\section{Data Availability}

The authors declare that data supporting the findings of this study are available within the article.

\section{References}

1. Schuppan D, Afdhal NH. Liver cirrhosis. Lancet. 2008;371(9615):838-851.

2. GBD 2017 Cirrhosis Collaborators. The global, regional, and national burden of cirrhosis by cause in 195 countries and territories, 1990-2017: a systematic analysis for the Global Burden of Disease Study 2017. Lancet Gastroenterol Hepatol. 2020;5(3):245-266.

3. Publications | National Institute on Alcohol Abuse and Alcoholism | Surveillance Report \#114 [Internet]. [cited Jun 9, 2021]. Available from: https://pubs.niaaa.nih.gov/ publications/surveillance114/Cirr17.htm.

4. Gross M. [Liver cirrhosis and the most common complications: diagnosis and treatment]. MMW Fortschr Med. 2015;157(Suppl 1):75-79.
5. Gordon FD. Ascites. Clin Liver Dis. 2012;16(2):285-299.

6. Arroyo V. Pathophysiology, diagnosis and treatment of ascites in cirrhosis. Ann Hepatol. 2002;1(2):72-79.

7. Garbuzenko DV, Arefyev NO. Current approaches to the management of patients with cirrhotic ascites. World J Gastroenterol. 2019;25(28):3738-3752.

8. Gines P, Fernandez-Esparrach G, Arroyo V, Rodes J. Pathogenesis of ascites in cirrhosis. Semin Liver Dis. 1997; 17(3):175-189.

9. Reichle FA, Owen OE. Hemodynamic patterns in human hepatic cirrhosis: a prospective randomized study of the hemodynamic sequelae of distal splenorenal (Warren) and mesocaval shunts. Ann Surg. 1979;190(4):523-534.

10. Urrunaga NH, Singal AG, Cuthbert JA, Rockey DC. Hemorrhagic ascites. Clinical presentation and outcomes in patients with cirrhosis. J Hepatol. 2013;58(6):11131118.

11. Stang A. Critical evaluation of the Newcastle-Ottawa scale for the assessment of the quality of nonrandomized studies in meta-analyses. Eur J Epidemiol. 2010;25(9):603605.

12. Liberati A, Altman DG, Tetzlaff J, Mulrow C, Gotzsche PC, Ioannidis JP, Clarke M, et al. The PRISMA statement for reporting systematic reviews and meta-analyses of studies that evaluate healthcare interventions: explanation and elaboration. BMJ. 2009;339:b2700.

13. DeSitter L, Rector WG. The significance of blood ascites in patients with cirrhosis. American Journal of Gastroenterology. 1984;79(2):136-138.

14. Yildiz H, Akdogan M, Suna N, Oztas E, Kuzu UB, Bilge Z, Aydinli O, et al. Cirrhosis with ascites: Is the presence of hemorrhagic ascites an indicator of poor prognosis? Turk J Gastroenterol. 2016;27(4):349-353.

15. Naqvi IH, Mahmood K, Talib A. Haemorrhagic versus non haemorrhagic ascites in cirrhosis: Their relationship and impact on prognosis of liver cirrhosis. Pak J Med Sci. 
2020;36(4):603-608.

16. Buob S, Johnston AN, Webster CR. Portal hypertension: pathophysiology, diagnosis, and treatment. J Vet Intern Med. 2011;25(2):169-186.

17. Filippi MD. Mechanism of diapedesis: importance of the transcellular route. Adv Immunol. 2016;129:25-53.
18. Akriviadis EA. Hemoperitoneum in patients with ascites. Am J Gastroenterol. 1997;92(4):567-575.

19. Okano J, Shiota G, Horie Y, Mitsuda A, Suou T, Kawasaki H, Oofuji S. Rupture of metastatic nodule on the peritoneal surface secondary to hepatocellular carcinoma. Intern Med. 1996;35(10):783-784. 